\title{
Persistent monoarthritis on the patient of parkinson's disease with cognitive impairment and frequent falls
}

\begin{abstract}
Parkinson's disease (PD) is often associated with cognitive impairment and frequent falls. Although the cause of frequent falls is not clearly understood, cognitive impairment is thought to be the cause of frequent falls and these frequent falls can lead to serious complications such as traumatic arthritis. Traumatic arthritis is a wellknown condition that develops in the joints as a result of recurrent trauma. Here we presented a 61-year-old female patient with PD who suffered from severe pain, limitation, swelling, temperature increase and erythema of right knee also difficulty of gait. In the result of all the detailed evaluations made, conservative treatment-resistant traumatic monoarthritis and osteonecrosis were diagnosed due to recurrent falls of the patient and the patient was directed to surgical treatment. The patient's falls were ongoing due to cognitive impairment and PD and she continued to suffer from pain and swelling despite performing unicompartmental (lateral compartment) knee arthroplasty. As a result of consultations with orthopaedists we decided to perform a total knee arthroplasty on the patient. After surgery, the patient was admitted to our rehabilitation clinic for orthopedic and PD rehabilitation program. During the rehabilitation program, the symptoms of swelling and pain in the patient's knee improved and the frequency of falls decreased. The patient's rehabilitation program still continues in our clinic.
\end{abstract}

Volume 2 Issue 5 - 2017

\author{
Canan Çelik,' Illker Illhanli,' Elif Uysal,' Adem \\ Türköz,' Fazil Kulakli,' Özkan Özen² \\ Department of Chalcolithic and the Bronze Age Archaeology, \\ The National Academy of Sciences of Ukraine, Ukraine
}

\begin{abstract}
Correspondence: Fazil Kulakli, Giresun University, Ilhan Özdemir Training and Research Hospital, Department of Physical Medicine and Rehabilitation, Giresun, Turkey, Email drfzl46@gmail.com
\end{abstract}

Received: November 21, 2017 | Published: December 22, 2017

\section{Introduction}

Parkinson's disease is often associated with cognitive impairment and frequent falls. ${ }^{1}$ Postural control is severely reduced in PD and postural instability is thought to be one of the main symptoms of the disease..$^{2-4}$ Walking speed is reduced in PD patients and this is accompanied by a decrease in arm swing. In addition, there is an increase in cadence, double support, and left-right gait asymmetry and there is a decrease in bilateral coordination. ${ }^{1}$ Gait disorders and postural instability increase the risk of falls in PD, which causes severe head trauma, fractures and mortality. ${ }^{1}$ In addition, levodopa-induced dyskinesia, symptomatic orthostatic hypotension, sudden loss of postural reflexes, coexisting neurological or other medical disorders, and environmental factors are the other risk factors for falls in PD. ${ }^{5,6}$ In the majority of the studies, the falls are related to the cognitive impairment. However, these studies evaluated advanced PD patients with gait disturbances and freezing problems. Cognitive disorders are observed from the early stages of the PD and may include several cognitive domains. Executive dysfunction and attention deficit are two of the most notable dysfunctions, and these are directly related to the risk of falls. ${ }^{7}$

After primary arthritis and rheumatoid arthritis, the third most common cause of total knee replacement is post-traumatic arthritis. Trauma can cause acute cartilage damage with necrosis of cartilage cells and chronic damage following alteration of the normal limb axis. Femoral and tibial fractures that regain leg deformity are other causes of cartilage damage. The deformity leads to chronic abnormal stress on joint surfaces and subsequent cartilage degeneration. ${ }^{8}$ In this study, we aimed to present a patient with PD who had recurrent falls and conservative treatment-resistant monoarthritis secondary to falls. We think that traumatic monoarthritis and recurrent falls in patients with
PD might improve by effective surgical treatment and rehabilitation programme when they do not respond to conservative treatment.

\section{Case report}

A 61-year-old female patient with PD who suffered from severe pain, limitation, swelling, temperature increase and erythema of right knee also difficulty of gait presented to our clinic. The patient could not walk for six months because of severe pain in her right knee. The patient had previously undergone joint aspiration, injection, and physical therapy and rehabilitation program. The patient indicated that she had been intermittently fallen formerly but the frequency of falls has been increased in the last 1 year. The pain was measured with Visual Analogue Scale (VAS 0 to 10) which is a measurement instrument that tries to measure a characteristic or attitude that is believed to range across a continuum of values and cannot easily be directly measured. ${ }^{9}$ The patient's pain was serious and the VAS score was 10. The pain was measured with Visual Analogue Scale (VAS 0 to 10) which is a measurement instrument that tries to measure a characteristic or attitude that is believed to range across a continuum of values and cannot easily be directly measured. ${ }^{9}$ The patient's pain was serious and the VAS score was 10 . There was a 20 -degree limit on knee extension at the physical examination of the patient. The patient's sitting balance was present but the standing balance was not present due to excessive pain. Pain in the knee was accompanied by erythema and swelling. The synovial fluid was aspirated twice and the knee was bandaged. Non-inflammatory synovial fluid findings were detected. CRP and erythrocyte sedimentation rate values were 0,56 and $11 \mathrm{~mm} / \mathrm{h}$ respectively and other laboratory findings were normal. On the knee Magnetic Resonance Imaging (MRI) examination; subchondral crescentic hyperintense signal changes in proton weighted images in the lateral condyle of the femur, and 
widespread subchondral hypointense signal changes in the T1 weighted images. The edematous signal in the lateral condyle of the femur was present. In addition, intra-articular and suprapatellar bursal fluid increase, patellar grade 1 chondromalasic changes, grade 3 tears in the lateral meniscus anterior horn and grade 2 degenerations in the lateral-medial meniscus posterior horns were observed in the images. Widespread hypodensities were observed in the lateral condyle of the femur in the knee Computer Tomography (CT) performed 50 days after the MRI examination. The patient's bilateral knee X-ray showed no significant pathology. Conclusion according to the FICAT classification $^{10}$ grade 2 osteonecrosis was accepted. The cognitive performance of the patient was examined with the Mini-Mental Test ${ }^{11}$ and the Montreal Cognitive Assessment Test ${ }^{12}$ and the scores were 25 and 17 respectively. Non-steroidal anti-inflammatory Drugs, steroids, analgesics and other conservative treatments such as physical therapy methods (Transcutaneous electrical nerve stimulation, cold pack) and exercises (Range of motion exercises, isometric and active assistive strengthening exercises, gentle stretching exercises, hip and ankle strengthening exercises etc.) were administered but there was no improvement in symptoms. Because the patient did not respond to conservative treatment, she was referred to the orthopedic clinic and decided to perform lateral compartment arthroplasty. After the operation, the patient was re-admitted to our clinic to start the physical therapy and rehabilitation program quickly. The patient's falls were ongoing due to cognitive impairment and PD and she continued to suffer from pain and swelling despite performing unicompartmental knee arthroplasty. As a result of consultations with orthopaedists, we decided to perform a total knee arthroplasty on the patient. After surgery, the patient was admitted to our rehabilitation clinic again and was scheduled for a total knee arthroplasty and PD rehabilitation program. During the rehabilitation program, significant improvement was detected in the joint range of motion, swelling and pain and the frequency of falls decreased. The loss of extension was improved from - 30 degrees to 0 , and flexion-60 degrees to-40, VAS was reduced from 10 to 3, and fall frequency was reduced from 5-6 times a day to 1-2 times a week. The patient's rehabilitation program still continues in our clinic. During the rehabilitation program, significant improvement was detected in the joint range of motion, swelling and pain and the frequency of falls decreased. The loss of extension was improved from-30 degrees to 0 , and flexion- 60 degrees to- 40 , VAS was reduced from 10 to 3 , and fall frequency was reduced from 5-6 times a day to 1-2 times a week. The patient's rehabilitation program still continues in our clinic. The patient's rehabilitation program still continues in our clinic (Figure 1) (Figure 2).
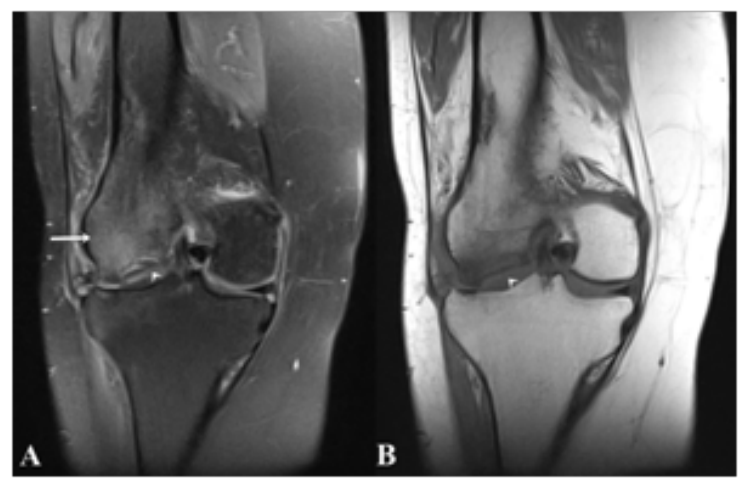

Figure I In the coronal fat-suppressed proton-weighted MR image (Figure IA), subchondral cresenteric hyperintense signal (arrowhead), edema in the femur lateral condyle (arrow) and subchondral hypointense signal changes (arrowhead) in the TI-weighted MR image (Figure IB) are observed.

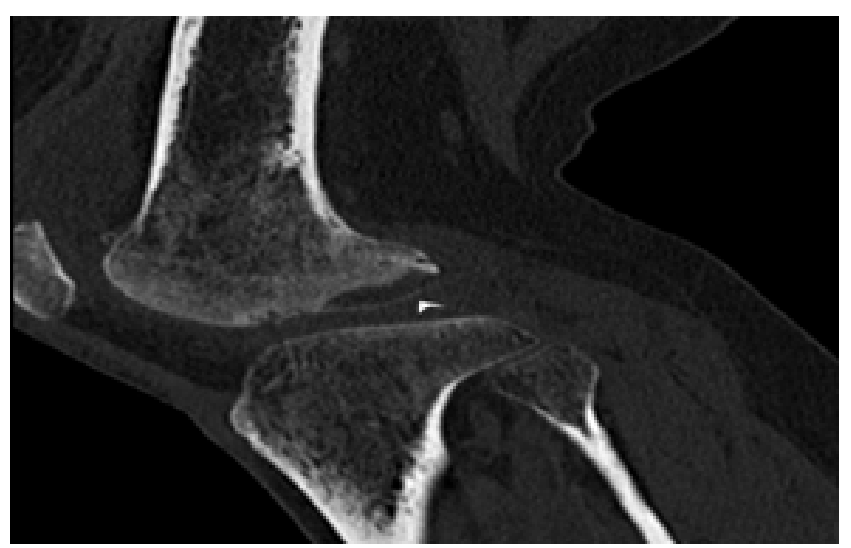

Figure 2 Sagittal reforming CT image shows a hypodense area (arrowhead) at the subchondral level in the lateral condyle of the femur.

\section{Conclusion}

Joint replacement surgery is an effective method in PD patients with conservative treatment-resistant chronic monoarthritis due to recurrent falls. Symptoms of patients improve after successful arthroplasty, resulting in decreased falls and increased effectiveness of rehabilitation programmes.

\section{Acknowledgements}

Special thanks to our patient giving consent for supporting the literature.

\section{Conflict of interest}

The author declares no conflict of interest.

\section{References}

1. Lauretani F, Galuppo L, Costantino C, et al. Parkinson's disease with dementia and falls is improved by AChEI? A preliminary study report. Aging Clin Exp Res. 2016;28(3):551-555.

2. Lauretani F, Maggio M, Silvestrini C, et al. Parkinson's disease (PD) in the elderly: an example of geriatric syndrome (GS). Arch Gerontol Geriatr. 2012;54(1):242-246.

3. Lauretani F, Ceda GP, Pelliccioni P, et al Approaching neurological diseases to reduce mobility limitations in older persons. Curr Pharm Des. 2014;20(19):3149-3164.

4. Vaugoyeau M, Viel S, Assaiante C, et al. Impaired vertical postural control and proprioceptive integration deficits in Parkinson's disease. Neuroscience. 2007;146(2):852-863.

5. Olanow CW, Watts RL, Koller WC. An algorithm (decision tree) for the management of Parkinson's disease: treatment guidelines. Neurology. 2001;56(Supp1 5):S1-88.

6. Michalowska M, Krygowska WA, Jedynecka U, et al. Analysis of the cause of falls in patients with Parkinson's disease. Neurol Neurochir Pol. 2002;36(1):57-68.

7. Kim JS, Jang W, Cho JW, et al. Bedside cognitive assessments and falls risk in Parkinson's disease. Neurol Sci. 2013;34(1):75-78.

8. Benazzo F, Rossi SMP, Combi A, et al. Knee replacement in chronic post-traumatic cases. EFORT Open Rev. 2016;1(5):211-218.

9. D Gould. Visual analogue scale (VAS). Journal of Clinical Nursing. 2001;10:697-706. 
10. Ficat RP, Arlet J. Necrosis of the femoral head. In: Hungerford DS, editor. Ischemia and necrosis of bone. Md: Williams \& Wilkins: USA;1980;171-182.

11. Folstein MF, Folstein SE, McHugh PR. Mini-mental state. A practical method for grading the cognitive state of patients for the clinician. $J$ Psychiatr Res. 1975;12(3):189-198.
12. Nasreddine ZS, Phillips NA, Bedirian V, et al. The montreal cognitive assessment, MoCA: A Brief Screening Tool For Mild Cognitive Impairment. 2005;53(4):695-699. 